\title{
ON THE RATIONAL COHOMOLOGY OF MODULI SPACES OF CURVES WITH LEVEL STRUCTURES
}

\author{
GILBERTO BINI AND CLAUDIO FONTANARI
}

\begin{abstract}
We investigate low degree rational cohomology groups of smooth compactifications of moduli spaces of curves with level structures. In particular, we determine $H^{k}\left(\bar{S}_{g}, \mathbb{Q}\right)$ for $g \geq 2$ and $k \leq 3$, where $\bar{S}_{g}$ denotes the moduli space of spin curves of genus $g$.
\end{abstract}

\section{INTRODUCTION}

Moduli spaces $M^{\Gamma}$ of curves with level structures are obtained by taking the quotient of the Teichmüller space by a finite index subgroup $\Gamma$ of the mapping class group. As such, they yield natural coverings of the moduli space $M_{g, n}$ of smooth genus $g$ curves with $n$ marked points.

The geometry of $M^{\Gamma}$ turns out to be better understood when $\Gamma$ contains the Torelli group, as explained in [8]. For instance, $\Gamma$ can be a spin mapping class group, so that $M^{\Gamma}$ is the moduli space $S_{g}^{ \pm}$parameterizing pairs (smooth genus $g$ curve $C$, even/odd theta characteristic on $C$ ). Under this assumption, a recent result by Putnam [12 shows that $H^{2}\left(M^{\Gamma}, \mathbb{Q}\right) \cong \mathbb{Q}$ for $g \geq 5$.

Here instead we investigate the rational cohomology of the canonical compactification $\bar{M}^{\Gamma}$ of $M^{\Gamma}$ over the moduli space $\bar{M}_{g, n}$ of stable curves. Indeed, the description of the boundary of $\bar{M}^{\Gamma}$ provided by [6] and [5] allows us to adapt the inductive approach introduced for $\bar{M}_{g, n}$ in [1] and recently refined in [2]. In particular, we are able to show that $H^{1}\left(\bar{M}^{\Gamma}, \mathbb{Q}\right)=0$ (see Theorem 2.3 ) and to determine a set of free generators for $\operatorname{Pic}\left(\bar{M}^{\Gamma}\right) \otimes \mathbb{Q}$ (see Theorem 4.1).

In the special case of spin moduli spaces, where a geometrically meaningful compactification has been constructed in [7], we obtain even stronger results. Namely, we get the vanishing of the third cohomology group (see Theorem 3.1) and a complete description of the second cohomology group (see Corollary 4.2).

Throughout, we work over the field $\mathbb{C}$ of complex numbers and all cohomology groups are intended to be with rational coefficients.

The first named author has been partially supported by "FIRST" Università di Milano and by MIUR Cofin 2008 - Varietà algebriche: geometria, aritmetica, strutture di Hodge. The second named author

2000 Mathematics Subject Classification: $14 \mathrm{H} 10$. 
has been partially supported by MIUR Cofin 2008 - Geometria delle varietà algebriche e dei loro spazi di moduli.

\section{The first Betti number of MOduli SPACES of CURVES WITH LEVEL STRUCTURES}

First, we recall some notation and basic facts on level structures: for more details, the reader is referred, for instance, to [6].

Let $\Sigma_{g, n}$ be a compact genus $g$ surface with $n$ marked points and let $\Gamma_{g, n}$ be its mapping class group. Denote by $T_{g, n}$ the Teichmüller space and by $M_{g, n}$ the moduli space $T_{g, n} / \Gamma_{g, n}$. A level $\Gamma$ is a subgroup of $\Gamma_{g, n}$. It is finite if $\Gamma$ has finite index in $\Gamma_{g, n}$. It is Galois if $\Gamma$ is normal in $\Gamma_{g, n}$. The functor of curves with $\Gamma$-level is represented by the analytic stack $\left[T_{g, n} / \Gamma\right]$, which is called a level structure over the moduli space $M_{g, n}$. Clearly, a level structure is a finite connected covering of $M_{g, n}$.

In what follows, we will focus on some particular level structures. Fix $g \geq 2$ and a basis of the first homology group of $\Sigma_{g, n}$ so that the intersection form is given by the $2 g \times 2 g$ matrix

$$
\left(\begin{array}{cc}
0 & I \\
-I & 0
\end{array}\right)
$$

There exists a surjective homomorphism $\Gamma_{g, n} \rightarrow S p(2 g, \mathbb{Z})$, where $S p(2 g, \mathbb{Z})$ is the symplectic group of $2 g \times 2 g$ matrices with integer entries. The kernel of this homomorphism is called the Torelli group $\mathcal{T}_{g, n}$. In some applications, we will take into account finite levels that contain the Torelli group. We briefly review some examples which fit into this picture.

Example A. For any integer $m \geq 2$, consider the surjective homomorphism $\Gamma_{g, n} \rightarrow S p(2 g, \mathbb{Z} / m \mathbb{Z})$ which maps an element $\gamma \in \Gamma_{g, n}$ to the homomorphism induced by $\gamma$ on the homology of $\Sigma_{g, n} \bmod m$. The kernel of this homomorphism is called the Abelian level of order $m$. For short, we will denote this level by $(m)$. By definition, it contains the Torelli group.

Those of Example A are particular examples of geometric levels. Indeed, if $\Pi^{\lambda}$ is a subgroup of the fundamental group $\Pi_{g, n}$ of $\Sigma_{g, n}$, then the geometric level determined by $\Pi^{\lambda}$ is the kernel $\Gamma^{\lambda}:=\operatorname{ker} \rho_{\lambda}$ of the natural representation:

$$
\left.\rho_{\lambda}: \Gamma_{g, n} \rightarrow \operatorname{Out}\left(\Pi_{g, n} / \Pi^{\lambda}\right)\right) .
$$

A level $\Gamma^{\lambda}$ is said to be fine if $\Gamma^{\lambda} \subset(m)$. In geometric terms, this means that $M^{\lambda}$ is a connected étale covering of $M^{(m)}$.

Example $B$. Let $V=H_{1}\left(\Sigma_{g, n}, \mathbb{Z} / 2 \mathbb{Z}\right)$ and let $\cdot$ denote the $\mathbb{Z} / 2 \mathbb{Z}$ intersection form on $V$. A $\mathbb{Z} / 2 \mathbb{Z}$-quadratic form on $\Sigma_{g, n}$ is a function $Q: V \rightarrow \mathbb{Z} / 2 \mathbb{Z}$ such that

$$
Q(x+y)=Q(x)+Q(y)+x \cdot y
$$


for all $x, y \in V$. The isomorphism class of $Q$ is determined by its Arf invariant. Given $Q$, let $G(Q)$ denote the subgroup of $\Gamma_{g, n}$ which preserves $Q$. Since there are two isomorphism classes of quadratic forms, there are two such groups, which are called the spin mapping class groups. It is well known that they contain the Torelli group. The space $T_{g, n} / G(Q)$ is the union of two connected components, $S_{g, n}^{+} \sqcup S_{g, n}^{-}$, which are called the moduli spaces of even (respectively, odd) spin structures.

A canonical compactification $\bar{M}^{\lambda}$ of $M^{\lambda}$ is obtained by taking the normalization of the Deligne-Mumford compactification $\bar{M}_{g, n}$ in the function field of $M^{\lambda}$. As proved in Proposition 1.6.8 in [5], if $\Gamma^{\lambda}$ is a finite fine level, then $\bar{M}^{\lambda}$ is represented by a projective variety.

Finally, we recall the geometry of the boundary as it is described in 6], Proposition 2.2 and Theorem 2.3, and in [5], Theorem 2.5.1 and Theorem 2.7.4. Let $\lambda$ be a fine geometric level structure over $M_{g, n}$ such that its compactification $\bar{M}^{\lambda}$ is smooth. An irreducible component of its Deligne-Mumford boundary, corresponding to reducible curves, is isomorphic to

$$
\bar{M}_{g_{1}, n_{1}+1}^{\lambda_{1}} \times \bar{M}_{g_{2}, n_{2}+1}^{\lambda_{2}}
$$

for some $g_{1}+g_{2}=g, n_{1}+n_{2}=n$ and with $\lambda_{i}$ suitably defined geometric levels (see [5], p. 25). Analogously, the closure of each stratum parameterizing singular irreducible curves is isomorphic to

$$
\bar{M}_{g-h, n+2 h}^{\lambda_{\sigma}},
$$

where $h \geq 1$ and $\lambda_{\sigma}$ is a suitably defined geometric level (see [5], p. $35)$.

In the special case of the spin mapping class group, a modular compactification à la Deligne-Mumford has been constructed by Cornalba in [7, where one can find an explicit description of the boundary as well (see [7], § 7).

Next, we turn to cohomological computations. Let $\Gamma$ be a level of $\Gamma_{g, n}$. We point out that, analogously to $M_{g, n}$, the homology of $M^{\Gamma}$ vanishes in high degree. In fact, the following holds.

Proposition 2.1. Let $\Gamma \subset \Gamma_{g, n}$. Then $H_{k}\left(M^{\Gamma}, \mathbb{Q}\right)=0$ for $k>c(g, n)$, where

$$
c(g, n)=\left\{\begin{array}{cc}
n-3 & g=0 \\
4 g-5 & g>0, n=0 \\
4 g-4+n & g>0, n>0
\end{array}\right.
$$

Proof. As proved in [9], Theorem 1.3, for every $n \geq 1$ there exists a $\Gamma_{g, n}$-equivariant homeomorphism of $T_{g, n}$ onto the arc complex. A fortiori, this is a $\Gamma$-equivariant homeomorphism. Thus, the standard proof of Harer's vanishing theorem [9] for the high degree homology of $M_{g, n}$ (see for instance [2], Lemma 2) shows that the same result holds 
for any such $M^{\Gamma}$ as well. On the other hand, the case $n=0$ is ruled out by a standard spectral sequence argument (we refer again to [2], last paragraph of Section 5, which adapts verbatim to our context).

As a consequence, we show how the inductive approach of [1] to the rational cohomology of $\bar{M}_{g, n}$ applies verbatim also to level structures and reduces the computation for a fixed degree to a few initial cases. In particular, for degree 1 the inductive basis is provided by the following easy fact.

Lemma 2.2. Denote by $\bar{M}^{\lambda}$ the smooth canonical compactification of a level structure of $\bar{M}_{0,4}$ or $\bar{M}_{1,1}$. Then $\bar{M}^{\lambda}$ is isomorphic to $\mathbb{P}^{1}$.

Proof. The degree $d$ covering map can be ramified only over points parameterizing curves with non-trivial automorphisms, whose number is 0 for $\bar{M}_{0,4}$ and 2 for $\bar{M}_{1,1}$ (see, for instance, [10, Corollary IV.4.7). Hence Hurwitz formula for the genus $g$ of $\bar{M}^{\Gamma}$ yields $2 g-2=d(-2)$ and $2 g-2 \leq d(-2)+2(d-1)$, respectively; in both cases it follows that $g=0$.

¿From Lemma 2.2, Proposition 2.1, Poincaré duality and the long exact sequence in cohomology with compact support

$$
\ldots \rightarrow H_{c}^{k}\left(M^{\lambda}\right) \rightarrow H^{k}\left(\bar{M}^{\lambda}\right) \rightarrow H^{k}\left(\partial \bar{M}^{\lambda}\right) \rightarrow \ldots
$$

we obtain the following result (see for instance [2], proof of Corollary 1 to Theorem 6).

Theorem 2.3. Let $\lambda$ be a fine geometric level structure over $M_{g, n}$ such that the canonical compactification $\bar{M}^{\lambda}$ over $\bar{M}_{g, n}$ is smooth. Then $H^{1}\left(\bar{M}^{\lambda}, \mathbb{Q}\right)=0$.

\section{LOW DEGREE COHOMOLOGY OF SPIN MODULI SPACES}

In the previous sections we have already mentioned the moduli space of spin curves constructed in [7]. More generally, for all integers $g, n$, $m_{1}, \ldots, m_{n}$, such that $2 g-2+n>0,0 \leq m_{i} \leq 1$ for every $i$, and 
$\sum_{i=1}^{n} m_{i}$ is even, one can consider the moduli spaces

$\bar{S}_{g, n}^{\left(m_{1}, \ldots, m_{n}\right)}:=\left\{\left[\left(C, p_{1}, \ldots, p_{n} ; \zeta ; \alpha\right)\right]:\left(C, p_{1}, \ldots, p_{n}\right)\right.$ is a genus $g$

quasi-stable projective curve with $n$ marked points;

$\zeta$ is a line bundle of degree $g-1+\frac{1}{2} \sum_{i=1}^{n} m_{i}$ on $C$

having degree 1 on every exceptional component of $C$, and $\alpha: \zeta^{\otimes 2} \rightarrow \omega_{C}\left(\sum_{i=1}^{n} m_{i} p_{i}\right)$ is a homomorphism which is not zero at a general point of every non-exceptional component of $C$ \}.

Here we prove the following result on the rational cohomology of $\bar{S}_{g, n}^{\left(m_{1}, \ldots, m_{n}\right)}$ :

Theorem 3.1. For every $g, n$ and $\left(m_{1}, \ldots, m_{n}\right)$ as above, we have

$$
H^{1}\left(\bar{S}_{g, n}^{\left(m_{1}, \ldots, m_{n}\right)}, \mathbb{Q}\right)=H^{3}\left(\bar{S}_{g, n}^{\left(m_{1}, \ldots, m_{n}\right)}, \mathbb{Q}\right)=0 .
$$

We are going to apply the inductive strategy developed by Arbarello and Cornalba in [1] for the moduli space of curves. Namely, we consider the long exact sequence of cohomology with compact supports:

(1)

$$
\ldots \rightarrow H_{c}^{k}\left(S_{g, n}^{\left(m_{1}, \ldots, m_{n}\right)}\right) \rightarrow H^{k}\left(\bar{S}_{g, n}^{\left(m_{1}, \ldots, m_{n}\right)}\right) \rightarrow H^{k}\left(\partial S_{g, n}^{\left(m_{1}, \ldots, m_{n}\right)}\right) \rightarrow \ldots
$$

Hence, whenever $H_{c}^{k}\left(S_{g, n}^{\left(m_{1}, \ldots, m_{n}\right)}\right)=0$, there is an injection $H^{k}\left(\bar{S}_{g, n}^{\left(m_{1}, \ldots, m_{n}\right)}\right)$ $\hookrightarrow H^{k}\left(\partial S_{g, n}^{\left(m_{1}, \ldots, m_{n}\right)}\right)$. Moreover, from [7], § 3, it follows that each irreducible component of the boundary of $\bar{S}_{g, n}^{\left(m_{1}, \ldots, m_{n}\right)}$ is the image of a morphism:

where either

$$
\mu_{i}: X_{i} \rightarrow \bar{S}_{g, n}^{\left(m_{1}, \ldots, m_{n}\right)}
$$

$$
X_{i}=\bar{S}_{a, s+1}^{\left(u_{1}, \ldots, u_{s+1}\right)} \times \bar{S}_{b, t+1}^{\left(v_{1}, \ldots, v_{t+1}\right)}
$$

with $a+b=g, s+t=n$, and $\sum_{i=1}^{s} u_{i}+\sum_{i=1}^{t} v_{i}=\sum_{i=1}^{n} m_{i}$; or

$$
X_{i}=\bar{S}_{g-1, n+2}^{\left(m_{1}, \ldots, m_{n}, m_{n+1}, m_{n+2}\right)} .
$$

Finally, exactly as in [1], Lemma 2.6, a bit of Hodge theory implies that the map

$$
H^{k}\left(\bar{S}_{g, n}^{\left(m_{1}, \ldots, m_{n}\right)}\right) \rightarrow \oplus_{i} H^{k}\left(X_{i}\right)
$$

is injective whenever $H^{k}\left(\bar{S}_{g, n}^{\left(m_{1}, \ldots, m_{n}\right)}\right) \rightarrow H^{k}\left(\partial S_{g, n}^{\left(m_{1}, \ldots, m_{n}\right)}\right)$ is. So we obtain the claim of Theorem 3.1 by induction, provided we show that $H_{c}^{1}\left(S_{g, n}^{\left(m_{1}, \ldots, m_{n}\right)}\right)=H_{c}^{3}\left(S_{g, n}^{\left(m_{1}, \ldots, m_{n}\right)}\right)=0$ for almost all the values of $g$, 
$n$, and we check that $H^{1}\left(\bar{S}_{g, n}^{\left(m_{1}, \ldots, m_{n}\right)}\right)=H^{3}\left(\bar{S}_{g, n}^{\left(m_{1}, \ldots, m_{n}\right)}\right)=0$ for all the remaining values of $g, n$.

¿From Proposition 2.1 and Poincaré duality we deduce that

$$
H_{c}^{1}\left(S_{g, n}^{\left(m_{1}, \ldots, m_{n}\right)}\right)=0
$$

for any $g \geq 2$, for $g=1, n \geq 2$, and for $g=0, n \geq 5$; and

$$
H_{c}^{3}\left(S_{g, n}^{\left(m_{1}, \ldots, m_{n}\right)}\right)=0
$$

for any $g \geq 3$, for $g=2, n \geq 2$, for $g=1, n \geq 4$, and for $g=0, n \geq 7$. Hence we have only to verify that

$$
\begin{aligned}
H^{1}\left(\bar{S}_{0, n}^{\left(m_{1}, \ldots, m_{n}\right)}\right) & =0, n \leq 4 \\
H^{3}\left(\bar{S}_{0, n}^{\left(m_{1}, \ldots, m_{n}\right)}\right) & =0, n \leq 6 \\
H^{1}\left(\bar{S}_{1,1}^{(m)}\right) & =0 \\
H^{3}\left(\bar{S}_{2}\right) & =0 \\
H^{3}\left(\bar{S}_{2,1}^{(m)}\right) & =0 \\
H^{3}\left(\bar{S}_{1, n}^{\left(m_{1}, \ldots, m_{n}\right)}\right) & =0, n \leq 3 .
\end{aligned}
$$

The first two checks are straightforward: since any two divisors of the same degree on $\mathbb{P}^{1}$ are linearly equivalent, there are natural isomorphisms

$$
\bar{S}_{0, n}^{\left(m_{1}, \ldots, m_{n}\right)} \cong \bar{M}_{0, n}
$$

Since $H^{k}\left(\bar{M}_{0, n}\right)=0$ for every odd $k$ by Keel's results (see [11]), (3) and (4) easily follow.

As for (5), first of all notice that $\bar{S}_{1,1}^{(1)}=\emptyset$ by degree reasons. Next, recall that $\bar{S}_{1,1}^{(0)}$ is the union of two connected components $\bar{S}_{1,1}^{(0),+}$ and $\bar{S}_{1,1}^{(0),-}$, corresponding respectively to even and odd spin structures. If $\left(E ; q_{1}\right)$ is a smooth 1-pointed elliptic curve, the linear series $\left|2 q_{1}\right|$ realizes $E$ as a two-sheeted covering of $\mathbb{P}^{1}$ branched at $q_{1}$ and at other three points $q_{2}, q_{3}$, and $q_{4}$. The curve $E$ carries one odd theta-characteristic $\left(L=\mathcal{O}_{E}\right)$, and three even ones (namely, $\mathcal{O}_{E}\left(q_{1}-q_{2}\right), \mathcal{O}_{E}\left(q_{1}-q_{3}\right)$, and $\left.\mathcal{O}_{E}\left(q_{1}-q_{4}\right)\right)$. The uniqueness of the odd theta-characteristic on $E$ implies the existence of a natural isomorphism

$$
\bar{S}_{1,1}^{(0),-} \cong \bar{M}_{1,1} \cong \mathbb{P}^{1},
$$

hence $H^{1}\left(\bar{S}_{1,1}^{(0),-}\right)=H^{1}\left(\mathbb{P}^{1}\right)=0$. Finally we turn to $\bar{S}_{1,1}^{(0),+}$. We claim that there is a surjective morphism

$$
f: \bar{M}_{0,4} \longrightarrow \bar{S}_{1,1}^{(0),+} \text {. }
$$

Indeed, let $\left(C ; p_{1}, p_{2}, p_{3}, p_{4}\right)$ be a 4 -pointed stable genus zero curve. The morphism $f$ associates to it the admissible covering $E$ of $C$ branched at 
the $p_{i}{ }^{\prime}$ 's, pointed at $q_{1}$ and equipped with the line bundle $\mathcal{O}_{E}\left(q_{1}-q_{2}\right)$, where $q_{i}$ denotes the point of $E$ lying above $p_{i}$. It follows that

$$
H^{1}\left(\bar{S}_{1,1}^{(0),+}\right) \hookrightarrow H^{1}\left(\bar{M}_{0,4}\right)=H^{1}\left(\mathbb{P}^{1}\right)=0
$$

and (5) is completely proved.

The proofs of (6) and (7) are similar. Again, $\bar{S}_{2,1}^{(1)}=\emptyset$ by degree reasons and $\bar{S}_{2, n}^{(0, \ldots, 0)}$ is the disjoint union of $\bar{S}_{2, n}^{(0, \ldots, 0),+}$ and $\bar{S}_{2, n}^{(0, \ldots, 0),-}$ Moreover, if $C$ is a smooth hyperelliptic curve and $q_{i}(i=1, \ldots, 6)$ are the ramification points of the hyperelliptic involution, then $C$ carries six odd theta-characteristics (namely, $\left.\mathcal{O}_{C}\left(q_{i}\right), i=1, \ldots, 6\right)$ and ten even ones (namely, $\mathcal{O}_{C}\left(q_{i}+q_{j}-q_{k}\right)$, with $i, j$ and $k$ distinct). We claim that there are surjective morphisms:

$$
\begin{aligned}
& f^{+}: \bar{M}_{0,6} \longrightarrow \bar{S}_{2}^{+} \\
& f^{-}: \bar{M}_{0,6} \longrightarrow \bar{S}_{2}^{-} \\
& g^{+}: \bar{M}_{0,7} \longrightarrow \bar{S}_{2,1}^{(0,0),+} \\
& g^{-}: \bar{M}_{0,7} \longrightarrow \bar{S}_{2,1}^{(0,0),-}
\end{aligned}
$$

In order to define $f^{+}$and $f^{-}$, let $\left(C ; p_{1}, \ldots p_{6}\right)$ be a 6 -pointed, stable, genus zero curve. The morphism $f^{+}$(respectively, $f^{-}$) associates to it the admissible covering $Y$ of $C$ branched at the $p_{i}$ 's and equipped with the line bundle $\mathcal{O}_{Y}\left(q_{1}+q_{2}-q_{3}\right)$ (respectively, $\mathcal{O}_{Y}\left(q_{1}\right)$ ), where $q_{i}$ denotes the point of $E$ lying above $p_{i}$. As for $g^{+}$and $g^{-}$, let $\left(C ; p_{1}, \ldots p_{7}\right)$ be a 7 -pointed stable genus zero curve. The morphism $g^{+}$(respectively, $g^{-}$) associates to it the admissible covering $Y$ of $C$ branched at the $p_{i}$ 's, pointed at one of the two points lying above $p_{7}$ (of course different choices produce isomorphic curves) and equipped with the line bundle $\mathcal{O}_{Y}\left(q_{1}+q_{2}-q_{3}\right)$ (respectively, $\mathcal{O}_{Y}\left(q_{1}\right)$ ), where $q_{i}$ denotes the point of $E$ lying above $p_{i}$. Hence we obtain injective maps in cohomology $H^{k}\left(\bar{S}_{2}^{+}\right) \hookrightarrow H^{k}\left(\bar{M}_{0,6}\right), H^{k}\left(\bar{S}_{2}^{-}\right) \hookrightarrow H^{k}\left(\bar{M}_{0,6}\right)$, $H^{k}\left(\bar{S}_{2,1}^{(0,0),+}\right) \hookrightarrow H^{k}\left(\bar{M}_{0,7}\right)$, and $H^{k}\left(\bar{S}_{2,1}^{(0,0),-}\right) \hookrightarrow H^{k}\left(\bar{M}_{0,7}\right)$, which reduce (6) and (7) to Keel's results mentioned above.

The proof of (8) turns out to be more involved. The case $m_{1}=$ $m_{2}=m_{3}=0$ has already been addressed in [4], Lemma 4 , so here we directly turn to $\bar{S}_{1,3}^{(1,1,0)}$. However, our inductive approach requires to handle $\bar{S}_{1,2}^{(1,1)}$ too.

The boundary components of $\bar{S}_{1,2}^{(1,1)}$ are the following:

- $A_{\text {irr }}$, whose general member is obtained from a smooth 4-pointed rational curve $C$ carrying the line bundle $\mathcal{O}_{C}(P)$ by collapsing two marked points in an ordinary node; 
- $B_{\text {irr }}$, whose general member is obtained from a smooth 4-pointed rational curve carrying the line bundle $\mathcal{O}_{C}$ by joining two marked points with an exceptional component;

- $A_{1, \emptyset}$, whose general member is obtained by joining with an exceptional component a smooth 1-pointed elliptic curve $(E, p)$ carrying an even root of $\mathcal{O}_{E}$ and a smooth 3-pointed rational curve $\left(C, q, p_{1}, p_{2}\right)$ carrying the line bundle $\mathcal{O}_{C}$;

- $B_{1, \emptyset}$, whose general member is obtained by joining with an exceptional component a smooth 1-pointed elliptic curve $(E, p)$ carrying the line bundle $\mathcal{O}_{E}$ and a smooth 3 -pointed rational curve $\left(C, q, p_{1}, p_{2}\right)$ carrying the line bundle $\mathcal{O}_{C}$.

Next, we list the boundary components of $\bar{S}_{1,3}^{(1,1,0)}$ :

- $A_{\text {irr }}$, whose general member is obtained from a smooth 5-pointed rational curve $C$ carrying the line bundle $\mathcal{O}_{C}(P)$ by collapsing two marked points in an ordinary node;

- $B_{\text {irr }}$, whose general member is obtained from a smooth 5-pointed rational curve carrying the line bundle $\mathcal{O}_{C}$ by joining two marked points with an exceptional component;

- $A_{1, \emptyset}$, whose general member is obtained by joining with an exceptional component a smooth 1-pointed elliptic curve $(E, p)$ carrying an even root of $\mathcal{O}_{E}$ and a smooth 4-pointed rational curve $\left(C, q, p_{1}, p_{2}, p_{3}\right)$ carrying the line bundle $\mathcal{O}_{C}$;

- $B_{1, \emptyset}$, whose general member is obtained by joining with an exceptional component a smooth 1-pointed elliptic curve $(E, p)$ carrying the line bundle $\mathcal{O}_{E}$ and a smooth 4-pointed rational curve $\left(C, q, p_{1}, p_{2}, p_{3}\right)$ carrying the line bundle $\mathcal{O}_{C}$;

- $\Delta_{1,\{1\}}$, whose general member is obtained by joining with an ordinary node a smooth 2-pointed elliptic curve $\left(E, p, p_{1}\right)$ carrying a square root of $\mathcal{O}_{E}\left(p+p_{1}\right)$ and a smooth 3 -pointed rational curve $\left(C, q, p_{2}, p_{3}\right)$ carrying the line bundle $\mathcal{O}_{C}$;

- $\Delta_{1,\{2\}}$, whose general member is obtained by joining with an ordinary node a smooth 2-pointed elliptic curve $\left(E, p, p_{2}\right)$ carrying a square root of $\mathcal{O}_{E}\left(p+p_{2}\right)$ and a smooth 3 -pointed rational curve $\left(C, q, p_{1}, p_{3}\right)$ carrying the line bundle $\mathcal{O}_{C}$;

- $A_{1,\{3\}}$, whose general member is obtained by joining with an exceptional component a smooth 2-pointed elliptic curve $\left(E, p, p_{3}\right)$ carrying an even root of $\mathcal{O}_{E}$ and a smooth 3-pointed rational curve $\left(C, q, p_{1}, p_{2}\right)$ carrying the line bundle $\mathcal{O}_{C}$;

- $B_{1,\{3\}}$, whose general member is obtained by joining with an exceptional component a smooth 2-pointed elliptic curve $\left(E, p, p_{3}\right)$ carrying the line bundle $\mathcal{O}_{E}$ and a smooth 3-pointed rational curve $\left(C, q, p_{1}, p_{2}\right)$ carrying the line bundle $\mathcal{O}_{C}$.

Lemma 3.2. The vector space $H^{2}\left(\bar{S}_{1,2}^{(1,1)}\right)$ is generated by boundary classes. 
Proof. We claim that the four boundary classes $\alpha_{\text {irr }}, \beta_{\text {irr }}, \alpha_{1, \emptyset}$, and $\beta_{1, \emptyset}$ are linearly independent. Indeed, suppose that there is a relation:

$$
a_{0} \alpha_{\mathrm{irr}}+b_{0} \beta_{\mathrm{irr}}+a_{1} \alpha_{1, \emptyset}+b_{1} \beta_{1, \emptyset}=0 .
$$

By restricting (10) to $B_{\text {irr }}, A_{\text {irr }}, B_{1, \emptyset}$ and $A_{1, \emptyset}$, we obtain $a_{1}=0, b_{1}=0$, $a_{0}=0$ and $b_{0}=0$, respectively. Since we already know that the first Betti number vanishes, it will be sufficient to prove that

$$
\chi\left(\bar{S}_{1,2}^{(1,1)}\right)=6 .
$$

We first compute $\chi\left(S_{1,2}^{(1,1)}\right)$. A point of $S_{1,2}^{(1,1)}$ corresponds to a smooth 2-pointed elliptic curve $\left(E ; p_{1}, p_{2}\right)$ together with a square root of the line bundle $\mathcal{O}_{E}\left(p_{1}+p_{2}\right)$, that is, a ramification point of the 2 -sheeted covering of $\mathbb{P}^{1}$ defined by the linear series $\left|p_{1}+p_{2}\right|$. Hence the natural projection $S_{1,2}^{(1,1)} \rightarrow M_{1,2}$ is generically four-to-one, but there are a few special fibers with less than four points. Indeed, consider the twosheeted covering of $\mathbb{P}^{1}$ defined by the linear series $\left|2 p_{1}\right|$ and ramified over $\infty, 0,1$, and $\lambda$, with $p_{1}$ lying above $\infty$. If $p_{2}$ lies above 0 , then the corresponding involution exchanges cyclically the square roots of $\mathcal{O}_{E}\left(p_{1}+p_{2}\right)$. If moreover $\lambda=-1$, then the projectivity of $\mathbb{P}^{1}$ defined by $z \mapsto-z$ induces another automorphism of $\left(E ; p_{1}, p_{2}\right)$ and in this case all square roots of $\mathcal{O}_{E}\left(p_{1}+p_{2}\right)$ are identified. Finally, if $\lambda=-\omega$ (with $\omega^{3}=1$ ) and $p_{2}$ is one point lying above $\frac{\omega}{\omega-1}$ then the projectivity of $\mathbb{P}^{1}$ defined by $z \mapsto \frac{z+\omega}{\omega}$ induces automorphisms of $\left(E, p_{1}, p_{2}\right)$ exchanging ciclically three square roots of $\mathcal{O}_{E}\left(p_{1}+p_{2}\right)$. As in [1], p. 124, we denote by $X$ the locus of all curves $\left(E ; p_{1}, p_{2}\right)$ such that $p_{2}$ is a 2-torsion point with respect to the group law with origin in $p_{1}$. Therefore, since $X$ is isomorphic to the quotient $M_{0,4}^{\prime}$ of $M_{0,4}$ modulo the operation of interchanging the labelling of two of the marked points, we have:

$$
\begin{aligned}
\chi\left(S_{1,2}^{(1,1)}\right)= & 4 \chi\left(M_{1,2} \backslash X \cup\{\text { point }\}\right)+2 \chi(X \backslash\{\text { point }\})+\chi(\text { point })+ \\
& +2 \chi(\text { point })=4 \chi\left(M_{1,2}\right)-2 \chi\left(M_{0,4}^{\prime}\right)-3=1
\end{aligned}
$$

(recall that $\chi\left(M_{0,4}^{\prime}\right)=0$ and $\chi\left(M_{1,2}\right)=1$ by [1], (5.3) and (5.4)).

Next, from the stratification of $\bar{M}_{1,2}$ by graph type (see [1], Figure 1), it follows that

$$
\chi\left(\bar{S}_{1,2}^{(1,1)}\right)=\chi\left(S_{1,2}^{(1,1)}\right)+2 \chi\left(M_{0,4}^{\prime}\right)+\chi\left(S_{1,1}^{(0),+}\right)+\chi\left(S_{1,1}^{(0),-}\right)+3+1 .
$$

Since $\chi\left(S_{1,1}^{(0),+}\right)=0$ by [4], $(5)$, and $\chi\left(S_{1,1}^{(0),-}\right)=\chi\left(M_{1,1}\right)=1$, we obtain $\chi\left(\bar{S}_{1,2}^{(1,1)}\right)=6$, as claimed.

The following result is a partial analogue of [4], Lemma 3.

Lemma 3.3. Let $x$ and $y$ be distinct and not belonging to $\{1,2,3\}$. Let $\mathfrak{S}_{2}$ be the symmetric group permuting $x$ and $y$. Define

$$
\xi: \bar{M}_{0,\{1,2,3, x, y\}} / \mathfrak{S}_{2} \longrightarrow B_{\mathrm{irr}} \hookrightarrow \bar{S}_{1,3}^{(1,1,0)}
$$


by joining the points labelled $x$ and $y$ with an exceptional component. Then the kernel of

$$
\xi^{*}: H^{2}\left(\bar{S}_{1,3}^{(1,1,0)}\right) \longrightarrow H^{2}\left(\bar{M}_{0,\{1,2,3, x, y\}} / \mathfrak{S}_{2}\right)
$$

is four-dimensional and generated by $\alpha_{\mathrm{irr}}, \beta_{\mathrm{irr}}, \beta_{1, \emptyset}$, and $\beta_{1,\{3\}}$.

Proof. It is clear that $\xi^{*}\left(\alpha_{\text {irr }}\right)=\xi^{*}\left(\beta_{1, \emptyset}\right)=\xi^{*}\left(\beta_{1,\{3\}}\right)=0$ because all the corresponding boundary divisors are disjoint from $B_{\text {irr }}$. Next, from $\xi^{*}\left(\alpha_{\text {irr }}\right)=0$ and [1], Lemma 3.16, it follows that also $\xi^{*}\left(\beta_{\text {irr }}\right)=0$. Conversely, if $\xi^{*}(\alpha)=0$ then we claim that for a suitable choice of rational coefficients $x, y, z, w$ the class $\gamma=\alpha-x \alpha_{\text {irr }}-y \beta_{\text {irr }}-z \beta_{1, \emptyset}-$ $w \beta_{1,\{3\}}$ vanishes on $\bar{S}_{1,3}^{(1,1,0)}$, hence the class $\alpha$ is a linear combination of $\alpha_{\text {irr }}, \beta_{\text {irr }}, \beta_{1, \emptyset}$, and $\beta_{1,\{3\}}$. In order to show that $\gamma=0$ we first check that its restriction to all boundary components vanishes and then we observe that the restriction map is injective in our case. More precisely, we define

$$
\zeta: \bar{M}_{0,\{1,2,3, x, y\}} / \mathfrak{S}_{2} \longrightarrow A_{\mathrm{irr}} \hookrightarrow \bar{S}_{1,3}^{(1,1,0)}
$$

by joining the points labelled $x$ and $y$ with an ordinary node.

Since $h^{2}\left(\bar{M}_{0,\{1,2,3, x, y\}} / \mathfrak{S}_{2}\right)=4$, for any class $\alpha \in H^{2}\left(\bar{S}_{1,3}^{(1,1,0)}\right)$ we have

$$
\zeta^{*}(\alpha)=h \zeta^{*}\left(\beta_{1, \emptyset}\right)+k \zeta^{*}\left(\beta_{1,\{3\}}\right)+s \zeta^{*}\left(\delta_{1,\{1\}}\right)+t \zeta^{*}\left(\delta_{1,\{2\}}\right)
$$

for some $h, k, s, t \in \mathbb{Q}$.

Suppose now $\xi^{*}(\alpha)=0$. Then the class $\beta:=\alpha-h \beta_{1, \emptyset}-k \beta_{1,\{3\}}$ satisfies $\zeta^{*}(\beta)=s \zeta^{*}\left(\delta_{1,\{1\}}\right)+t \zeta^{*}\left(\delta_{1,\{2\}}\right)$ and $\xi^{*}(\beta)=0$. If

$$
\begin{aligned}
\rho: H^{2}\left(\bar{S}_{1,3}^{(1,1,0)}\right) \longrightarrow & H^{2}\left(A_{\text {irr }}\right) \oplus H^{2}\left(B_{\text {irr }}\right) \oplus H^{2}\left(A_{1, \emptyset}\right) \oplus H^{2}\left(B_{1, \emptyset}\right) \oplus \\
& H^{2}\left(\Delta_{1,\{1\}}\right) \oplus H^{2}\left(\Delta_{1,\{2\}}\right) \oplus H^{2}\left(A_{1,\{3\}}\right) \oplus H^{2}\left(B_{1,\{3\}}\right)
\end{aligned}
$$

is the restriction to the boundary components, we have:

$$
\begin{aligned}
\rho(\beta)= & \left(\zeta^{*}(\beta), 0,\left(a \delta_{\mathrm{irr}}, 0\right), \beta \cdot B_{1, \emptyset}, c_{1} \alpha_{\mathrm{irr}}+c_{2} \beta_{\mathrm{irr}}+c_{3} \alpha_{1, \emptyset}+c_{4} \beta_{1, \emptyset},\right. \\
& \left.d_{1} \alpha_{\mathrm{irr}}+d_{2} \beta_{\mathrm{irr}}+d_{3} \alpha_{1, \emptyset}+d_{4} \beta_{1, \emptyset}, e \delta_{\mathrm{irr}}, \beta \cdot B_{1,\{3\}}\right),
\end{aligned}
$$

where cohomology classes are expressed in standard bases for the second cohomology groups of the moduli spaces dominating the various boundary components. In particular, we have $a \delta_{\text {irr }} \in H^{2}\left(\bar{S}_{1,1}^{(0),+}\right)$, $c_{1} \alpha_{\text {irr }}+c_{2} \beta_{\text {irr }}+c_{3} \alpha_{1, \emptyset}+c_{4} \beta_{1, \emptyset} \in H^{2}\left(\bar{S}_{1,2}^{(1,1)}\right), d_{1} \alpha_{\text {irr }}+d_{2} \beta_{\text {irr }}+d_{3} \alpha_{1, \emptyset}+$ $d_{4} \beta_{1, \emptyset} \in H^{2}\left(\bar{S}_{1,2}^{(1,1)}\right), e \delta_{\text {irr }} \in H^{2}\left(\bar{S}_{1,2}^{(0,0),+}\right)$. The vanishing of several coefficients is due to the fact that all the above classes restrict to zero on $B_{\text {irr }}$ since $\xi^{*}(\alpha)=0$. For instance, from Lemma 3.2 it follows that $H^{2}\left(\bar{S}_{1,2}^{(1,1)}\right)$ is generated by $\alpha_{\text {irr }}, \beta_{\text {irr }}, \alpha_{1, \emptyset}$, and $\beta_{1, \emptyset}$. Under the corresponding morphism

$$
\xi^{\prime}: \bar{M}_{0,\{1,2, x, y\}} / \mathfrak{S}_{2} \longrightarrow B_{\text {irr }} \hookrightarrow \bar{S}_{1,2}^{(1,1)}
$$

the class $\alpha_{1, \emptyset}$ pulls back to $\delta_{0,\{x, y\}}$, which is not zero, so the kernel of $\xi^{*}$ is generated by $\alpha_{\text {irr }}, \beta_{\text {irr }}$, and $\beta_{1, \emptyset}$, and we have $c_{3}=d_{3}=0$. 
Moreover, the various restrictions have to coincide also on all the other overlaps between boundary components. In particular, a careful case-by-case direct inspection shows the following implications:

- $A_{\text {irr }} \cap \Delta_{1,\{1\}} \neq \emptyset \Rightarrow s=c_{4}=c_{3}=0$;

- $A_{\text {irr }} \cap \Delta_{1,\{2\}} \neq \emptyset \Rightarrow t=d_{4}=d_{3}=0$;

- $B_{1, \emptyset} \cap A_{\text {irr }} \neq \emptyset \Rightarrow \beta \cdot B_{1, \emptyset}=\left(b \delta_{\text {irr }}, 0\right)$;

- $B_{1,\{3\}} \cap A_{\text {irr }} \neq \emptyset \Rightarrow \beta . B_{1,\{3\}}=f \delta_{\text {irr }}$;

- $A_{1,\{3\}} \cap A_{1, \emptyset} \neq \emptyset \Rightarrow e=a$;

- $B_{1,\{3\}} \cap B_{1, \emptyset} \neq \emptyset \Rightarrow f=b$;

- $\Delta_{1,\{1\}} \cap A_{1, \emptyset} \neq \emptyset \Rightarrow c_{1}+c_{2}=a$;

- $\Delta_{1,\{1\}} \cap B_{1, \emptyset} \neq \emptyset \Rightarrow c_{1}=b$;

- $\Delta_{1,\{2\}} \cap A_{1, \emptyset} \neq \emptyset \Rightarrow d_{1}+d_{2}=a$;

- $\Delta_{1,\{2\}} \cap B_{1, \emptyset} \neq \emptyset \Rightarrow d_{1}=b$.

As a consequence, if

$$
\gamma:=\beta-b \alpha_{\mathrm{irr}}-(a-b) \beta_{\mathrm{irr}}=\alpha-b \alpha_{\mathrm{irr}}-(a-b) \beta_{\mathrm{irr}}-h \beta_{1, \emptyset}-k \beta_{1,\{3\}}
$$

then

$$
\rho(\gamma)=(0,0,(0,0),(0,0), 0,0,0,0) .
$$

On the other hand, Proposition 2.1 with $k=4, g=1, n=3$, and the inductive argument following (1) imply that $\rho$ is injective, hence $\gamma=0$ and our claim is proved.

Lemma 3.4. The vector space $H^{2}\left(\bar{S}_{1,3}^{(1,1,0)}\right)$ is generated by boundary classes.

Proof. Let $V$ the subspace of $H^{2}\left(\bar{S}_{1,3}^{(1,1,0)}\right)$ generated by the pull-backs $\delta_{1, \emptyset}, \delta_{1,\{1\}}, \delta_{1,\{2\}}$, and $\delta_{1,\{3\}}$ of the corresponding boundary divisors on $\bar{M}_{1,3}$. In view of Lemma 3.3 , it will be sufficient to show that the morphism $\xi^{*}$ vanishes modulo $V$. In order to do so, we adapt the argument in [1], pp. 114-118. If $\alpha$ is any class in $H^{2}\left(\bar{S}_{1,3}^{(1,1,0)}\right)$, then

$\xi^{*}(\alpha)=a_{\{x, y\}} \delta_{0,\{x, y\}}+a_{\{1,3\}} \delta_{0,\{1,3\}}+a_{\{2,3\}} \delta_{0,\{2,3\}}+a_{\{3\}}\left(\delta_{0,\{x, 3\}}+\delta_{0,\{y, 3\}}\right)$.

The idea is simply to modify $\alpha$ with elements of $V$ in such a way that $\xi^{*}(\alpha)=0$. The first move consists in adding to $\alpha$ a suitable multiple of $\delta_{1, \emptyset}$ so as to make $a_{\{x, y\}}=0$. Next, by Lemma 3.2 there exists a suitable multiple of $\delta_{1,\{3\}}$ which added to $\alpha$ makes $a_{\{3\}}=0$ (for details, see [1], p. 116). Finally, the third move consists in adding to $\alpha$ a suitable linear combination of $\delta_{1,\{1\}}$ and $\delta_{1,\{2\}}$ so as to make $a_{\{1,3\}}=a_{\{2,3\}}=0$. Hence we obtain $\xi^{*}(\alpha)=0$, as desired.

Finally, we conclude the proof of (8) .

Lemma 3.5. We have $H^{3}\left(\bar{S}_{1,3}^{(1,1,0)}\right)=0$. 
Proof. By Lemma 3.4, $H^{2}\left(\bar{S}_{1,3}^{(1,1,0)}\right)$ is generated by the eight boundary classes $\alpha_{\text {irr }}, \beta_{\text {irr }}, \alpha_{1, \emptyset}, \beta_{1, \emptyset}, \delta_{1,\{1\}}, \delta_{1,\{2\}}, \alpha_{1,\{3\}}$, and $\beta_{1,\{3\}}$. Hence $h^{2}\left(\bar{S}_{1,3}^{(1,1,0)}\right) \leq 8$; next, we claim that

$$
\chi\left(\bar{S}_{1,3}^{(1,1,0)}\right)=18
$$

hence $h^{3}\left(\bar{S}_{1,3}^{(1,1,0)}\right)=0$.

First of all, we compute $\chi\left(S_{1,3}^{(1,1,0)}\right)$. The natural projection $S_{1,3}^{(1,1,0)} \rightarrow$ $M_{1,3}$ is generically four-to-one, but there are a few special fibers with less than four points. Indeed, denote by $Y$ the locus of all curves $\left(E ; p_{1}, p_{2}, p_{3}\right)$ such that both $p_{2}$ and $p_{3}$ are 2 -torsion points with respect to the group law with origin in $p_{1}$. Then it is clear that the fiber of $p$ over $Y$ consists of two points; moreover, if $\left(E ; p_{1}, p_{2}, p_{3}\right)$ is the 2 -sheeted covering of $\mathbb{P}^{1}$ ramified over $\infty, 0,1$, and $-\omega$ (with $\omega^{3}=1$ ), with $p_{1}$ lying above $\infty$ and $p_{2}, p_{3}$ lying above $\frac{\omega}{\omega-1}$, then the projectivity of $\mathbb{P}^{1}$ defined by $z \mapsto \frac{z+\omega}{\omega}$ induces automorphisms of $\left(E ; p_{1}, p_{2}, p_{3}\right)$ exchanging cyclically three square roots of $\mathcal{O}_{E}\left(p_{2}+p_{3}\right)$. Next, we claim that $\chi(Y)=$ 0 . Indeed, it is clear that $M_{0,4} \backslash$ \{point is a 2-sheeted covering of $Y \backslash\{$ point $\}$. Hence

$$
\chi(Y)=\frac{\chi\left(M_{0,4} \backslash\{\text { point }\}\right)}{2}+1=0,
$$

as claimed. It follows that

$$
\chi\left(S_{1,3}^{(1,1,0)}\right)=4 \chi\left(M_{1,3} \backslash Y \cup\{\text { point }\}\right)+2 \chi(Y)+2 \chi(\text { point })=-2
$$

(recall that $\chi\left(M_{1,3}\right)=0$ by [1], (5.4)).

Next, from the stratification of $\bar{M}_{1,3}$ by graph type (see [1], Figure 2) we obtain

$$
\begin{aligned}
\chi\left(\bar{S}_{1,3}^{(1,1,0)}\right)= & \chi\left(S_{1,3}^{(1,1,0)}\right)+2 \chi\left(M_{0,5}^{\prime}\right)+\chi\left(S_{1,1}^{(0),+}\right) \chi\left(M_{0,4}\right)+ \\
& +\chi\left(S_{1,1}^{(0),-}\right) \chi\left(M_{0,4}\right)+2 \chi\left(S_{1,2}^{(1,1)}\right)+\chi\left(S_{1,2}^{(0,0),+}\right)+ \\
& +\chi\left(S_{1,2}^{(0,0),-}\right)+3 \chi\left(M_{0,4}\right)+12 \chi\left(M_{0,4}^{\prime}\right)+3 \chi\left(S_{1,1}^{(0),+}\right)+ \\
& +3 \chi\left(S_{1,1}^{(0),-}\right)+9+5+2 .
\end{aligned}
$$

Here $M_{0,4}^{\prime}$ and $M_{0,5}^{\prime}$ denote the quotient of $M_{0,4}$ and $M_{0,5}$, respectively, modulo the operation of interchanging the labelling of two of the marked points; by [1], (5.3), we have $\chi\left(M_{0,4}^{\prime}\right)=0$ and $\chi\left(M_{0,5}^{\prime}\right)=1$. Moreover, we have $\chi\left(S_{1,1}^{(0),+}\right)=0, \chi\left(S_{1,1}^{(0),-}\right)=1, \chi\left(S_{1,2}^{(1,1)}\right)=1$ (see above, proof of Lemma 3.2), $\chi\left(S_{1,2}^{(0,0),+}\right)=0$ by [4], (6), $\chi\left(S_{1,2}^{(0,0),-}\right)=$ $\chi\left(M_{1,2}\right)=1$ by [1], (5.4), $\chi\left(M_{0,4}\right)=-1$, hence $\chi\left(\bar{S}_{1,3}^{(1,1,0)}\right)=18$, as desired. 


\section{The Picard group and the Second Cohomology group}

Let $\Gamma$ be a finite index subgroup of the mapping class group $\Gamma_{g, n}$ that contains the Torelli group. For instance, $\Gamma$ can be any of the levels in Example A or Example B in Section 2,

We recall that the Hodge class is defined to be the first Chern class $\lambda:=c_{1}\left(\pi_{*} \omega_{\pi}\right)$, where $\omega_{\pi}$ is the relative dualizing sheaf of the universal curve $\pi$. For any level $\Gamma$ denote by $p_{\Gamma}: \bar{M}^{\Gamma} \rightarrow \bar{M}_{g, n}$ the map induced by the definition of $\bar{M}^{\Gamma}$. Define on $\bar{M}^{\Gamma}$ the Hodge class to be the pull-back $p_{\Gamma}^{*}(\lambda)$, which we still denote by $\lambda$ by abuse of notation.

The universal cotangent classes of $\bar{M}_{g, n}$ are the first Chern classes of the line bundles $\mathcal{L}_{i}$ for $i=1, \ldots, n$, where the fiber of $\mathcal{L}_{i}$ over a pointed stable curve $\left[C ; x_{1}, \ldots, x_{n}\right]$ is the cotangent space $T_{x_{i}}^{*}(C)$ - we recall that the marked points of any stable curve are smooth. Naturally, the pull-backs under $p_{\Gamma}^{*}$ of the classes $\psi_{i}$ 's define universal cotangent classes on any level structure $M^{\Gamma}$.

Finally, the irreducible components of the Deligne-Mumford boundary of a level structure yield degree two cohomology classes via (rational) Poincaré duality. We refer to them as boundary classes.

This said, we can prove the following theorem.

Theorem 4.1. Let $\Gamma$ be a finite-index subgroup of the mapping class group that contains the Torelli group. Let $\bar{M}^{\Gamma}$ be the corresponding covering over $\bar{M}_{g, n}$. If $g \geq 5$, then $\operatorname{Pic}\left(\bar{M}^{\Gamma}\right) \otimes \mathbb{Q}$ is freely generated by the Hodge class, the set of $\psi$ classes, and the set of boundary classes.

Proof. From [12, Theorem 2.1, it follows that $H^{2}\left(M^{\Gamma}\right)$ is freely generated by the Hodge class and the set of $\psi$ classes. On the other hand, by [8], Theorem 5.4, we have $H^{2}\left(M^{\Gamma}, \mathbb{Q}\right) \cong \operatorname{Pic}\left(M^{\Gamma}\right) \otimes \mathbb{Q}$, hence we need only to check that the boundary classes are linearly independent. Indeed, by the proof of Theorem 10 in [2] $H^{1}\left(\bar{M}^{\Gamma}\right)=0$ implies that $H^{1}\left(M^{\Gamma}\right)$ is the kernel of the Gysin map $H^{0}\left(\partial \bar{M}^{\Gamma}\right) \rightarrow H^{2}\left(\bar{M}^{\Gamma}\right)$. Since by [8], Proposition 5.2, we have $H^{1}\left(M^{\Gamma}\right)=0$, it follows that there are no linear relations among boundary classes in $H^{2}\left(\bar{M}^{\Gamma}\right)$, hence the claim holds. In the special case of spin moduli spaces, the linear independence of boundary classes can be also checked directly by intersecting them with suitable test families, see [7], Proposition (7.2).

Now we focus on the special case of $\bar{S}_{g, n}^{\left(m_{1}, \ldots, m_{n}\right)}$. As pointed out in [3], the injection (2) is compatible with the Hodge decomposition. As a consequence, Proposition 2.1 and Poincaré duality imply that $h^{2,0}\left(\bar{S}_{g, n}^{\left(m_{1}, \ldots, m_{n}\right)}\right)=0$ for every $g, n$ if $h^{2,0}\left(\bar{S}_{0, n}^{\left(m_{1}, \ldots, m_{n}\right)}\right)=0, n \leq 5$, and $h^{2,0}\left(\bar{S}_{1, n}^{\left(m_{1}, \ldots, m_{n}\right)}\right)=0, n \leq 2$. On the other hand, $H^{2}\left(\bar{S}_{0, n}^{\left(m_{1}, \ldots, m_{n}\right)}\right)$ is algebraic by (9) and [11]. Moreover, $H^{2}\left(\bar{S}_{1, n}^{(0, \ldots, 0)}\right)$ is also algebraic by 
4], Proposition 2, and the same is true for $H^{2}\left(\bar{S}_{1,2}^{(1,1)}\right)$ by Lemma 3.2. Hence $h^{2,0}\left(\bar{S}_{g, n}^{\left(m_{1}, \ldots, m_{n}\right)}\right)$ vanishes and from the exponential sequence

$$
0 \rightarrow \mathbb{Z} \rightarrow \mathcal{O} \rightarrow \mathcal{O}^{*} \rightarrow 0
$$

we deduce that

$$
H^{2}\left(\bar{S}_{g, n}^{\left(m_{1}, \ldots, m_{n}\right)}\right) \cong H^{1}\left(\bar{S}_{g, n}^{\left(m_{1}, \ldots, m_{n}\right)}, \mathcal{O}^{*}\right)=\operatorname{Pic}\left(\bar{S}_{g, n}^{\left(m_{1}, \ldots, m_{n}\right)}\right) .
$$

In particular, the following holds.

Corollary 4.2. If $g \geq 5$ then $H^{2}\left(\bar{S}_{g, n}^{\left(m_{1}, \ldots, m_{n}\right)}\right)$ is freely generated by the Hodge class, the set of $\psi$ classes, and the set of boundary classes.

\section{REFERENCES}

[1] E. Arbarello, M. Cornalba: Calculating cohomology groups of moduli spaces of curves via algebraic geometry. Inst. Hautes Études Sci. Publ. Math. 88 (1998), $97-127$.

[2] E. Arbarello, M. Cornalba: Divisors in the moduli space of curves. Pre-print arXiv:0810.5373, to appear in Surveys in Differential Geometry.

[3] G. Bini, C. Fontanari: Moduli of curves and spin structures via algebraic geometry. Trans. Amer. Math. Soc. 358 (2006), 3207-3217.

[4] G. Bini, C. Fontanari: A remark on the rational cohomology of $\bar{S}_{1, n}$. Collectanea Mathematica 60 (2009), 241-247.

[5] M. Boggi: Compactifications of moduli spaces of curves, Ph.D. Dissertation, University of Utrecht, 1998.

[6] M. Boggi, M. Pikaart: Galois covers on moduli of curves. Comp. Math. 120 (2000), 171-191.

[7] M. Cornalba: Moduli of curves and theta characteristics. Lectures on Riemann surfaces (Trieste, 1987), 560-589, Teaneck, NJ: World Sci. Publishing, 1989.

[8] R. M. Hain: Torelli groups and geometry of moduli spaces of curves. Current topics in complex algebraic geometry, 97-143, Cambridge University Press, 1995.

[9] J. Harer: The virtual cohomological dimension of the mapping class group of an orientable surface. Inv. Math. 84 (1986), 157-176.

[10] R. Hartshorne: Algebraic geometry. Springer, 1977.

[11] S. Keel: Intersection theory of moduli space of stable $n$-pointed curves of genus zero. Trans. Amer. Math. Soc. 330 (1992), 545-574.

[12] A. Putman: The second rational homology group of the moduli space of curves with level structures. Pre-print arXiv:0809.4477.

E-mail address: gilberto.bini@unimi.it

Current address: Dipartimento di Matematica, Universitì degli Studi

di Milano, Via C. Saldini 50, 20133 Milano, Italy.

E-mail address: fontanar@science.unitn.it

Current address: Dipartimento di Matematica, Università Degli Studi di Trento, Via Sommarive 14, 38123 Trento, Italy. 